ANALELE ŞTIINŢIFICE ALE UNIVERSITĂŢII “AL.I. CUZA” DIN IAŞI (S.N.)

MATEMATICA, Tomul LXI, 2015, f.1

DOI: $10.2478 /$ aicu-2013-0047

\title{
A TAUBERIAN THEOREM FOR A GENERAL SUMMABILITY METHOD
}

\author{
BY
}

\author{
İBRAHIM ÇANAK
}

\begin{abstract}
We investigate conditions under which $M_{\varphi}$ summability implies Abel summability and give the generalized Littlewood Tauberian theorem for $M_{\varphi}$ summability method.

Mathematics Subject Classification 2010: 40E05, 40G10.

Key words: Tauberian theorems, power series methods, slow oscillation, Abel summability method, $M_{\varphi}$ summability method.
\end{abstract}

\section{Introduction}

Let $\mathcal{A}$ denote the space of analytic functions in $0<x<1$. To each $f(a)=f(a, x)=\sum_{n=0}^{\infty} a_{n} x^{n}$ in $\mathcal{A}$ we associate a series $\sum_{n=0}^{\infty} a_{n}$ of Taylor coefficients of $f$. A series $\sum_{n=0}^{\infty} a_{n}$ is said to be Abel summable to $s$ if $\sum_{n=0}^{\infty} a_{n} x^{n}$ converges for $0<x<1$ and tends to $\mathrm{s}$ as $x \rightarrow 1^{-}$. Abel's continuity theorem (see [1]) for power series states that if $\sum_{n=0}^{\infty} a_{n}$ converges, then $\lim _{x \rightarrow 1^{-}} f(a, x)=\sum_{n=0}^{\infty} a_{n}$. It is known that the converse of this implication is not true in general. However, we have a conditional converse of Abel's continuity theorem known as the generalized Littlewood Tauberian theorem (see [10]) for the Abel summability method asserting that if $\sum_{n=0}^{\infty} a_{n}$ is Abel summable to $s$ and slowly oscillating, then $\sum_{n=0}^{\infty} a_{n}=s$. We remind the reader that a series $\sum_{n=0}^{\infty} a_{n}$ is said to be slowly oscillating (see [7]) if

$$
\sum_{k=m+1}^{n} a_{k}=o(1), n>m \rightarrow \infty, n / m \rightarrow 1
$$


For a different proof of the generalized Littlewood Tauberian theorem (see [10]), we refer the reader to Landau [6], Schmidt [7], and ÇAnak [2].

Denote the class of kernels of the integral transforms of functions in $\mathcal{A}$ by $\Phi$. We now need the following properties of functions $\varphi$ in $\Phi$ :

1. There exists a number $\alpha_{0}=\alpha_{0}(\Phi) \in(0,1)$ such that every $\varphi \in \Phi$ is analytical in $\left[\alpha_{0}, 1\right)$.

2. For every $\varphi \in \Phi, \varphi(x) \rightarrow \infty, x \rightarrow 1^{-}$.

3. Each $\varphi \in \Phi$ is zero-free in $\left[\alpha_{0}, 1\right)$.

4. For each $m \geq 1, \frac{\varphi_{m}(x)}{\varphi_{m-1}(x)}=o(1), x \rightarrow 1^{-}$where $\varphi_{0}=\varphi$ and $\varphi_{m}(x)=$ $\int_{\alpha_{0}}^{x} \varphi_{m-1}(t) d t$.

For every $f$ in $\mathcal{A}$ and $\varphi \in \Phi$ we define $M(f, \varphi)=M(f, \varphi, x)=\frac{\int_{\alpha_{0}}^{x} f(t) \varphi(t) d t}{\varphi_{1}(x)}$ if $x \neq \alpha_{0}$ and $\lim _{x \rightarrow \alpha_{0}} M(f, \varphi, x)=f\left(\alpha_{0}\right)$ if $x=\alpha_{0}$.

A series $\sum_{n=0}^{\infty} a_{n}$ is said to be $M_{\varphi}$ summable to $s$ if

$$
\lim _{x \rightarrow 1^{-}} M(f, \varphi, x)=s .
$$

Example 1.1. The series $\sum_{n=0}^{\infty} a_{n}$ whose general term is the Taylor coefficient of the function $\mathrm{g}$ defined by $g(x)=f^{\prime}\left(\varphi_{1}(x)\right)$, where $f$ is bounded in $\mathcal{A}$ is $M_{\varphi}$ summable.

It is plain that every Abel summable series is $M_{\varphi}$ summable, but the converse statement is not always true. The $M_{\varphi}$ summability method is regular with respect to the Abel summability method. Indeed, since $\varphi_{1}^{\prime}(x)=\varphi(x)$, it easily follows that Abel summability of $\sum_{n=0}^{\infty} a_{n}$ implies $M_{\varphi}$ summability of $\sum_{n=0}^{\infty} a_{n}$. However, if a function $f$ satisfies the condition

$$
\frac{\int_{\alpha_{0}}^{x} f^{\prime}(t) \varphi_{1}(t) d t}{\varphi_{1}(x)}=o(1), x \rightarrow 1^{-},
$$

then the converse statement is also true. This follows from the identity

$$
f(x)=M(f, \varphi, x)+\frac{\int_{\alpha_{0}}^{x} f^{\prime}(t) \varphi_{1}(t) d t}{\varphi_{1}(x)} .
$$

An important subclass of $\Phi$ is the class of following functions $\psi_{m}(x)=$ $\frac{1}{(1-x)^{m}}, m=1,2, \ldots$ and $\alpha_{0}=0$. The function $\psi_{m}(x)=\frac{1}{(1-x)^{m}}$ defines a summability method $(A, m)$ which is regular with respect to Abel summability method. For the more information about the $M_{\varphi}$ and $(A, m)$ summability methods, we refer to [8]. Recently, a number of authors including 
ÇANAK ET AL. [3, 5], ÇANAK [4], and Totur [9] have given Tauberian theorems for the $(A, m)$ summability method.

The $(A, 1)$ summability method as a special case of $M_{\varphi}$ summability method is of considerable interest. A series $\sum_{n=0}^{\infty} a_{n}$ is said to be $(A, 1)$ summable to $s$ if

$$
\lim _{x \rightarrow 1^{-}} \frac{(1-x)}{x} \int_{0}^{x} \frac{f(t)}{(1-t)^{2}} d t=s .
$$

It is clear that Abel summability of $\sum_{n=0}^{\infty} a_{n}$ implies $(A, 1)$ summability of $\sum_{n=0}^{\infty} a_{n}$. That the converse is not true in general follows from the series $\sum_{n=0}^{\infty} a_{n}$ whose general term is the Taylor coefficient of the function $f$ defined by $f(x)=\sin \left((1-x)^{-1}\right)$ on $0<x<1$.

The identity (1.2) becomes

$$
f(x)=\frac{(1-x)}{x} \int_{0}^{x} \frac{f(t)}{(1-t)^{2}} d t+\frac{(1-x)}{x} \int_{0}^{x} \frac{f^{\prime}(t)}{1-t} d t
$$

for $\varphi(x)=\frac{1}{(1-x)^{2}}$. Since a series which is Abel summable to $s$ is $(\mathrm{A}, 1)$ summable to $s$, we have

$$
\frac{(1-x)}{x} \int_{0}^{x} \frac{f^{\prime}(t)}{1-t} d t=o(1), x \rightarrow 1^{-} .
$$

In the case where the condition (1.4) holds, every $(\mathrm{A}, 1)$ summable series is Abel summable.

An example of an $(A, 1)$ summable series can be obtained by applying integration by parts to $\frac{(1-x)}{x} \int_{o}^{x} \frac{f(t)}{(1-t)^{2}} d t$.

Example 1.2. If $\sum_{n=0}^{\infty} \frac{1}{n+1} \sum_{k=0}^{n} a_{k}$ is Abel summable to $s$, then $\sum_{n=0}^{\infty} a_{n}$ is $(A, 1)$ summable to 0 .

It is natural to ask under which conditions $M_{\varphi}$ summability implies Abel summability of $\sum_{n=0}^{\infty} a_{n}$. In this paper we both answer this question and give the generalized Littlewood Tauberian theorem for $M_{\varphi}$ summability method.

\section{A Tauberian theorem}

By the following theorem, we prove that every $M_{\varphi}$ summable series is Abel summable under certain conditions. 
Theorem 2.1. Let $\sum_{n=0}^{\infty} a_{n}$ be $M_{\varphi}$ summable to $s$. If

$$
\frac{\int_{\alpha_{0}}^{x} f^{\prime \prime}(t) \varphi_{2}(t) d t}{\varphi_{1}(x)}=o(1), x \rightarrow 1^{-}
$$

and $f$ has a zero of order three at $\alpha_{0}$ such that $\frac{f\left(\alpha_{0}\right)}{\varphi_{2}\left(\alpha_{0}\right)}$ is defined to be the limit $\lim _{x \rightarrow \alpha_{0}} \frac{f(x)}{\varphi_{2}(x)}$, then $\sum_{n=0}^{\infty} a_{n}$ is Abel summable to $s$.

Proof. Applying integration by parts to $M(f, \varphi, x)$, we have

$$
M(f, \varphi, x)=f(x)-T(f, \varphi, x),
$$

where $T(f, \varphi, x)=\frac{\int_{\alpha_{0}}^{x} f^{\prime}(t) \varphi_{1}(t) d t}{\varphi_{1}(x)}$. Applying again integration by parts to $T(f, \varphi, x)$, we have

$$
T(f, \varphi, x)=\frac{\varphi_{2}(x)}{\varphi_{1}(x)} f^{\prime}(x)-\frac{\int_{\alpha_{0}}^{x} f^{\prime \prime}(t) \varphi_{2}(t) d t}{\varphi_{1}(x)} .
$$

Combining (2.2) with (2.3), we obtain

$$
M(f, \varphi, x)=g(x)+\frac{\int_{\alpha_{0}}^{x} f^{\prime \prime}(t) \varphi_{2}(t) d t}{\varphi_{1}(x)},
$$

where $g(x)=f(x)-\frac{\varphi_{2}(x)}{\varphi_{1}(x)} f^{\prime}(x)$. Since $\sum_{n=0}^{\infty} a_{n}$ is $M_{\varphi}$ summable to $s$, we have by (2.1) and (2.4) that

$$
\lim _{x \rightarrow 1^{-}} g(x)=\lim _{x \rightarrow 1^{-}} M(f, \varphi, x)=s .
$$

Multiplying $g(x)$ by $\varphi_{1}(x)$ and dividing by $\varphi_{2}^{2}(x)$, we arrive at

$$
g(x)=-\frac{\varphi_{2}^{2}(x)}{\varphi_{1}(x)}\left(\frac{f(x)}{\varphi_{2}(x)}\right)^{\prime} .
$$

Since $\frac{f\left(\alpha_{0}\right)}{\varphi_{2}\left(\alpha_{0}\right)}$ is defined to be the limit $\lim _{x \rightarrow \alpha_{0}} \frac{f(x)}{\varphi_{2}(x)}$ at $\alpha_{0}$ where $f$ has a zero of order three, we obtain

$$
f(x)=-\varphi_{2}(x) \int_{\alpha_{0}}^{x} g(t) \frac{\varphi_{1}(t)}{\varphi_{2}^{2}(t)} d t .
$$


Since $\lim _{x \rightarrow 1^{-}} g(x)=s$, we have by L'Hospital's rule that

$$
\lim _{x \rightarrow 1^{-}} f(x)=s .
$$

This completes the proof.

Taking $\varphi(x)=\frac{1}{(1-x)^{2}}$ in Theorem 2.1, we have the following Tauberian theorem for the $(A, 1)$ summability method.

Corollary 2.2. Let $\sum_{n=0}^{\infty} a_{n}$ be $(A, 1)$ summable to $s$. If

$$
(1-x) \int_{0}^{x} \ln (1-t) f^{\prime \prime}(t) d t=o(1), x \rightarrow 1^{-}
$$

and $f$ has a zero of order three at 0 such that $\frac{f(x)}{-x-\ln (1-x))}$ at 0 is defined to be the limit $\lim _{x \rightarrow 0} \frac{f(x)}{-x-\ln (1-x)}$, then $\sum_{n=0}^{\infty} a_{n}$ is Abel summable to s.

We note that Theorem 2.1 can be given for the composition of $M_{\varphi}$ and $M_{\psi}$ defined by

$$
\left(M_{\psi} \circ M_{\varphi}\right)(f(a), x)=M\left(M_{\varphi}(f(a)), \psi, x\right)=\frac{\int_{\alpha_{0}}^{x} M(f, \varphi, t) \psi(t) d t}{\psi_{1}(x)},
$$

where $\varphi, \psi \in \phi$.

The following theorem is a consequence of the generalized Littlewood Tauberian theorem for Abel summability method.

Theorem 2.3. Let $\sum_{n=0}^{\infty} a_{n}$ be $M_{\varphi}$ summable to $s,(2.1)$ be satisfied and $f$ have a zero of order three at $\alpha_{0}$ such that $\frac{f\left(\alpha_{0}\right)}{\varphi_{2}\left(\alpha_{0}\right)}$ is defined to be the limit $\lim _{x \rightarrow \alpha_{0}} \frac{f(x)}{\varphi_{2}(x)}$. If $\sum_{n=0}^{\infty} a_{n}$ is slowly oscillating, then $\sum_{n=0}^{\infty} a_{n}$ converges to $s$.

Proof. We have by Theorem 2.1 that $\sum_{n=0}^{\infty} a_{n}$ is Abel summable to $s$. Since $\sum_{n=0}^{\infty} a_{n}$ is slowly oscillating, we have from the generalized Littlewood Tauberian theorem that $\sum_{n=0}^{\infty} a_{n}$ converges to $s$.

If the condition (2.1) is replaced by a stronger condition, we have the following result.

Theorem 2.4. Let $\sum_{n=0}^{\infty} a_{n}$ be $M_{\varphi}$ summable to $s$. If

$$
f^{\prime \prime}(x)=o\left(\frac{\varphi(x)}{\varphi_{2}(x)}\right), x \rightarrow 1^{-}
$$

and $\sum_{n=0}^{\infty} a_{n}$ is slowly oscillating, then $\sum_{n=0}^{\infty} a_{n}$ converges to $s$. 
Proof. We easily see that the condition (2.11) implies (2.1). It follows by Theorem 2.1 that $\sum_{n=0}^{\infty} a_{n}$ is Abel summable to $s$. Since $\sum_{n=0}^{\infty} a_{n}$ is slowly oscillating, we have from the generalized Littlewood Tauberian theorem that $\sum_{n=0}^{\infty} a_{n}$ converges to $s$.

\section{REFERENCES}

1. ABeL, N.H. - Recherches sur la série $1+\frac{m}{1} x+\frac{m(m-1)}{1.2} x^{2}+\frac{m(m-1)(m-2)}{1.2 .3} x^{3}+\ldots$, J. Reine Angew. Math., 1 (1826), 311-339.

2. ÇANAK, I. - A short proof of the generalized Littlewood Tauberian theorem, Appl. Math. Lett., 23 (2010), 818-820

3. ÇAnak, I.; Totur, Ü.; Dik, M. - One-sided Tauberian conditions for $(A, k)$ summability method, Math. Comput. Modelling, 51 (2010), 425-430.

4. ÇANAK, I. - A note on a Tauberian theorem for $(A, i)$ limitable method II, J. Comput. Anal. Appl., 13 (2011), 892-898.

5. ÇANAK, I.; Totur, Ü.; Dik, M. - On Tauberian theorems for $(A, k)$ summability method, Math. Slovaca, 61 (2011), 993-1001.

6. LANDAU, E. - Über einen satz des herrn Littlewood, Rend. Palermo., 35 (1913), 265276.

7. Schmidt, R. - Über divergente Folgen und lineare Mittelbildungen, Math. Z., 22 (1925), 89-152.

8. Stanojević, C.V.; Canak, I.; Stanojević, V.B. - Tauberian theorems for generalized abelian summability methods, Analysis of divergence (Orono, ME, 1997), 13-26, Appl. Numer. Harmon. Anal., Birkhäuser Boston, Boston, MA, 1999.

9. Totur, Ü.; ÇANAK, I.; DIK, M. - Some one-sided conditions under which subsequential convergence follows from $(A, k)$ summability method, Appl. Math. Lett., 24 (2011), 692-696.

10. Vijayaraghavan, T. - A Tauberian theorem, J. London Math. Soc., 1 (1926), 113120.

Received: 17.II.2012

Revised: 8.V.2012

Accepted: 10.V.2012
Ege University, Department of Mathematics, 35100, Izmir, TURKEY ibrahim.canak@ege.edu.tr 